\title{
Dynamic particle tracking reveals the ageing temperature of a colloidal glass
}

\author{
PING WANG, CHAOMING SONG AND HERNÁN A. MAKSE* \\ Levich Institute and Physics Department, City College of New York, New York 10031, USA \\ *e-mail: hmakse@levdec.engr.ccny.cuny.edu
}

T he glass transition ${ }^{1}$ is considered to be one of the most fundamental problems in statistical physics. Despite decades of effort, a general consensus on the validity of a universal theory for the large variety of glass systems is lacking ${ }^{2,3}$ - partly because of difficulties encountered in the experimental testing of the theoretical predictions ${ }^{4,5}$. Here, we present experiments on a colloidal glass made of micrometresized particles in a fluid. We investigate the autocorrelation and response function to monitor the ageing of a colloidal glass. At equilibrium, all the observables are stationary, whereas in the out-of-equilibrium glassy state they have an explicit dependence on the age of the system. We find that the transport coefficients scale with the ageing time as a power law, a signature of the slow relaxation. Nevertheless, our analysis reveals that the glassy system has thermalized at a constant temperature independent of the age and warmer than the bath, reflecting the structural rearrangements of cage dynamics. Furthermore, we find a universal scaling law to describe the global and local fluctuations of the observables.

Increasing the volume fraction of a colloidal system slows down the brownian dynamics of its constitutive particles, implying a limiting density, $\phi_{\mathrm{g}}$, above which the system can no longer be equilibrated with its bath ${ }^{1}$. Hence, the thermal system falls out of equilibrium on the timescale of the experiment and thus undergoes a glass transition ${ }^{2}$. Even above $\phi_{\mathrm{g}}$ the particles continue to relax, but the nature of the relaxation is very different to that in equilibrium. This phenomenon of a structural slow evolution beyond the glassy state is known as 'ageing's. The system is no longer stationary and the relaxation time is found to increase with the age of the system, $t_{\mathrm{w}}$, as measured from the time of sample preparation.

This picture applies not only to structural glasses such as colloids, silica and polymer melts, but also to spin glasses, ferromagnetic coarsening, elastic manifolds in quenched disorder and jammed matter such as grains and emulsions ${ }^{2,4,5}$. Theories originally developed in the field of spin glasses ${ }^{6}$ attempt to develop a common framework for the understanding of ageing. For example, the structural glass and spin-glass transitions have been coupled by the low-temperature extension of the modecoupling theory $y^{4,5,7}$. More generally, this approach is related to analogous ideas developed in the field of granular matter such as compactivity $^{8-11}$, and the inherent structure formalisms ${ }^{12}$, adapted to the energy landscape of glasses ${ }^{13}$.
One of the important features of this scenario is a separation of timescales where the observables are equilibrated at different temperatures, even though the system is far from equilibrium. Although theoretical results have flourished, the difficulties in the experimental testing of the fundamental predictions of the theories have hampered the development of an understanding of ageing in glasses ${ }^{14-18}$. Experiments so far have shown conflicting results, which are usually masked by large intermittent fluctuations in the observables ${ }^{17}$; a behaviour which seems beyond the current theoretical formalisms ${ }^{2}$. On the other hand, some numerical results are more favourable ${ }^{19-21}$. Furthermore, the concept of temperature has been shown to be useful to describe other far from equilibrium systems such as non-thermal granular materials ${ }^{9-11}$.

Here we use a model glass that is one of the simplest systems undergoing a glass transition: a colloidal glass of micrometresized particles, where the interactions between particles can be approximated as hard core potentials ${ }^{1,22,23}$. The system is index matched to allow the visualization of tracer particles in the microscope ${ }^{23}$. Owing to the simplicity of the system, we are able to follow the trajectories of magnetic tracers embedded in the colloidal sample and use this information as an ideal 'thermometer' to measure the temperature for the different modes of relaxation. In turn, we measure the autocorrelation function of the displacements and the integrated response to an external magnetic field as an indicator of the dynamics via a fluctuation-dissipation theorem (FDT). For this system we show that, even though the diffusivities and mobilities of the tracers scale with the age of the system, there is an effective temperature which is uniquely defined and remains constant independent of the age. This effective temperature is higher than the bath temperature and controls the slow relaxation of the system, as if the system was at 'equilibrium'. We find a scaling behaviour with the waiting time, which describes, in a unified way, not only the global but also the local fluctuations of the correlations and responses as well as the cage dynamics in the system.

\section{CORRELATIONS AND RESPONSES}

Our experiments use a colloidal suspension consisting of a mixture of poly(methylmethacrylate) sterically stabilized colloidal particles (radius $a_{\mathrm{p}}=1.5 \mu \mathrm{m}$, density $\rho_{\mathrm{p}}=1.19 \mathrm{~g} \mathrm{~cm}^{-3}$, polydispersity 
$\sim 14 \%$ ) plus a small fraction of superparamagnetic beads (radius $a_{\mathrm{m}}=1.6 \mu \mathrm{m}$ and density $\rho_{\mathrm{m}}=1.3 \mathrm{~g} \mathrm{~cm}^{-3}$, from Dynal Biotech) as the tracers (see the Methods section for further details). To investigate the dynamical properties of the ageing regime, we first consider the autocorrelation function as the mean square displacement (MSD) averaged over 82 tracer particles, $C\left(t, t_{\mathrm{w}}\right) \equiv$ $\left\langle\Delta x^{2}\left(t, t_{\mathrm{w}}\right)\right\rangle / 2=\left\langle\left[x\left(t_{\mathrm{w}}+\Delta t, t_{\mathrm{w}}\right)-x\left(t_{\mathrm{w}}, t_{\mathrm{w}}\right)\right]^{2}\right\rangle / 2$, at a given observation time, $t=t_{\mathrm{w}}+\Delta t$, after the sample has been ageing for $t_{\mathrm{w}}$ as measured from the end of the stirring process (see Supplementary Information, Appendix C). Then, we measure the integrated response function (by adding the external magnetic force, $F)$ given by the average position of the tracers, $\chi\left(t, t_{\mathrm{w}}\right) \equiv$ $\left\langle x\left(t_{\mathrm{w}}+\Delta t, t_{\mathrm{w}}\right)-x\left(t_{\mathrm{w}}, t_{\mathrm{w}}\right)\right\rangle / F$.

Analytical extensions of the mode-coupling theory for supercooled liquids to the low-temperature regime of glasses allow for the interpretation of the ageing of global correlation and response functions ${ }^{2,5}$. In these frameworks, the evolution of $C\left(t, t_{\mathrm{w}}\right)$ and $\chi\left(t, t_{\mathrm{w}}\right)$ are separated into a stationary part (short time) and an ageing part (long time): $C\left(t, t_{\mathrm{w}}\right)=C_{\mathrm{st}}\left(t-t_{\mathrm{w}}\right)+$ $C_{\mathrm{ag}}\left(t, t_{\mathrm{w}}\right)$ and $\chi\left(t, t_{\mathrm{w}}\right)=\chi_{\mathrm{st}}\left(t-t_{\mathrm{w}}\right)+\chi_{\mathrm{ag}}\left(t, t_{\mathrm{w}}\right)$, where we have included the explicit dependence on $t_{\mathrm{w}}$ in the ageing part.

This result can be rationalized in terms of the so-called 'cage dynamics'. As the density of the system increases the particles are trapped in cages. The motion inside the cage is still equilibrated at the bath temperature and is determined by the Gibbs distribution of states. The dynamics give rise to the stationary part of the response and correlation functions, which satisfy the usual equilibrium relations such as the FDT. However, the correlation does not decay to zero but remains constant because particles are trapped in cages for a long time. Thermally activated motions lead to a second structural relaxation that is responsible for the ageing part of the dynamics. In this regime the system is off equilibrium, and correlations and responses depend not only on the time of observation $t$ but also on the waiting time, $t_{\mathrm{w}}$. Slow, nonexponential relaxation ensues and a violation of the FDT is expected. However, this breakdown leads to a new definition of temperature for the slow modes, which has been proposed to be the starting point of a unifying description of ageing in glassy systems ${ }^{2}$.

Figure 1a shows $\left\langle\Delta x^{2}\left(t, t_{\mathrm{w}}\right)\right\rangle$ as a function of $\Delta t$ at a fixed $t_{\mathrm{w}}=100 \mathrm{~s}$ calculated for the three colloidal samples at $\phi_{\mathrm{C}}<\phi_{\mathrm{g}}<$ $\phi_{\mathrm{A}}<\phi_{\mathrm{B}}$, and Fig. 1b shows the age dependence for the glassy sample A. Sample B shows $t_{\mathrm{w}}$ dependence similar to sample A, whereas sample $\mathrm{C}$, being at equilibrium, is independent of $t_{\mathrm{w}}$, that is, it is stationary (see Supplementary Information, Appendix E).

The cage dynamics are evinced by the plateau observed in the MSD in the two glassy samples. The rattling of particles inside the cages is too fast $\left(\sim 10^{-2} \mathrm{~s}\right)^{24}$ to be observed with our visualization capabilities. As we focus mainly on the long-relaxation-time regime, the stationary parts, $C_{\mathrm{st}}$ and $\chi_{\mathrm{st}}$, are negligible compared with $C_{\mathrm{ag}}$ and $\chi_{\mathrm{ag}}$, in the following we concentrate only on the ageing part of the observables and drop the subscript ag: $C=C_{\mathrm{ag}}$ and $\chi=\chi_{\text {ag }}$. The tracers' motion is confined by the cage, which persists for a time of the order of the relaxation time $\tau\left(t_{\mathrm{w}}\right)$. As expected for an ageing system, this relaxation time increases with $t_{\mathrm{w}}$ as observed in Fig. 1b. For longer times, $\Delta t>\tau\left(t_{\mathrm{w}}\right)$, structural rearrangements lead to a second increase of the MSD, defining a diffusion regime characterized by a diffusion constant, $D\left(t_{\mathrm{w}}\right)$, which depends on the waiting time. We find an asymptotic form:

$$
\left\langle\left[x\left(t_{\mathrm{w}}+\Delta t, t_{\mathrm{w}}\right)-x\left(t_{\mathrm{w}}, t_{\mathrm{w}}\right)\right]^{2}\right\rangle \sim 2 D\left(t_{\mathrm{w}}\right) \Delta t, \text { for } \Delta t>\tau\left(t_{\mathrm{w}}\right) .
$$

Figure 1c shows the average displacement of the magnetic beads under the external force as a function of time $\Delta t$, for various ageing times, $t_{\mathrm{w}}$, in sample $\mathrm{A}$. The magnetic force is set as small as possible to observe the linear-response regime, $F=1.7 \times 10^{-14} \mathrm{~N}$
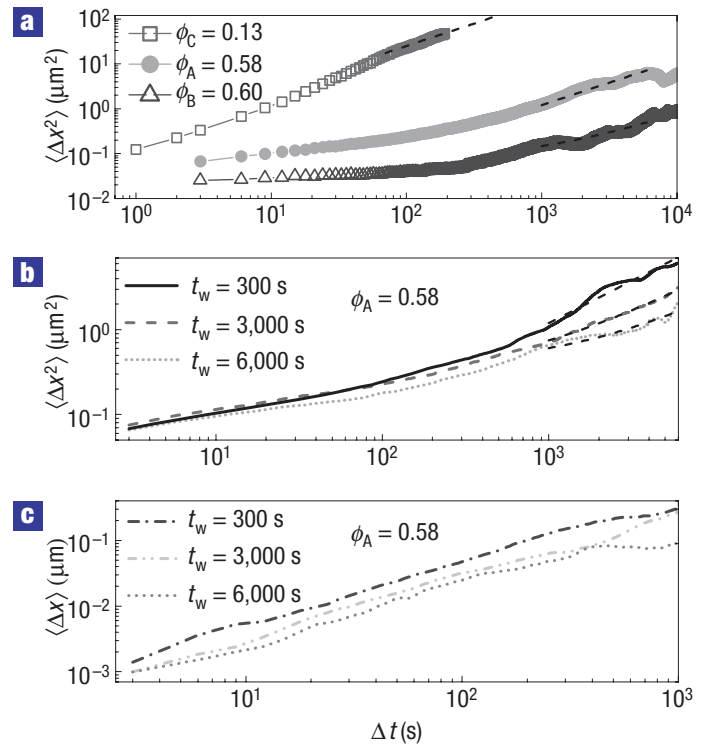

Figure 1 Autocorrelation and response functions. a, Mean square displacement of tracers as a function of $\Delta t$ for the three samples $\phi_{\mathrm{C}}=0.13<\phi_{\mathrm{g}}<\phi_{\mathrm{A}}=0.58$ $<\phi_{\mathrm{B}}=0.60$ at $t_{\mathrm{w}}=100 \mathrm{~s}$. The dashed straight lines indicate estimates for the diffusivity $D\left(t_{\mathrm{w}}\right)$. $\mathbf{b}$, Mean square displacement of tracers in sample $\mathrm{A}$ as a function of time $\Delta t$, for various ageing times $t_{\mathrm{w}}$. The dashed straight lines indicate the fitting regime to calculate the diffusivity $D\left(t_{\mathrm{w}}\right)$. c, Mean displacement of tracers under the magnetic force in sample $\mathrm{A}$ for various ageing times $t_{\mathrm{w}}$.

(see Supplementary Information, Appendix F). In contrast to the behaviour of the MSD, the integrated response function does not show the plateau characteristic of the cage effect. The data can be fitted to:

$$
\left\langle x\left(t_{\mathrm{w}}+\Delta t, t_{\mathrm{w}}\right)-x\left(t_{\mathrm{w}}, t_{\mathrm{w}}\right)\right\rangle \sim M\left(t_{\mathrm{w}}\right) F \Delta t,
$$

where $M\left(t_{\mathrm{w}}\right)$ is the mobility of the tracers, which is again waitingtime dependent as seen in the figure.

To investigate the nature of the scaling behaviour of the ageing regime, we study the dynamical behaviour with respect to $t_{\mathrm{w}}$. Figure 2 shows both the diffusivity and mobility as a function of $t_{\mathrm{w}}$ for sample A. Both $D\left(t_{\mathrm{w}}\right)$ and $M\left(t_{\mathrm{w}}\right)$ decrease with $t_{\mathrm{w}}$ signalling the slowing down in the dynamics. More importantly, they decrease according to a power law with the same exponent for both quantities:

$$
D\left(t_{\mathrm{w}}\right) \propto t_{\mathrm{w}}^{-\gamma} \text { and } M\left(t_{\mathrm{w}}\right) \propto t_{\mathrm{w}}^{-\gamma},
$$

with $\gamma=0.32 \pm 0.08$. This result is consistent with previous work in a similar ageing colloidal system, where it was found that the MSD $\left\langle\Delta x^{2}\right\rangle$ has a power-law decay with $t_{\mathrm{w}}$ (ref. 25). The fact that all the quantities scale as power laws indicates that the ageing regime lasts for a very long time, perhaps without ever equilibrating.

\section{EFFECTIVE TEMPERATURE}

The same power-law decay of the diffusivity and mobility implies that the system has thermalized at a constant effective temperature $T_{\text {eff }}$ independent of $t_{\mathrm{w}}$. This temperature is given by an extension of the Stokes-Einstein relation or FDT to out-of-equilibrium systems. 


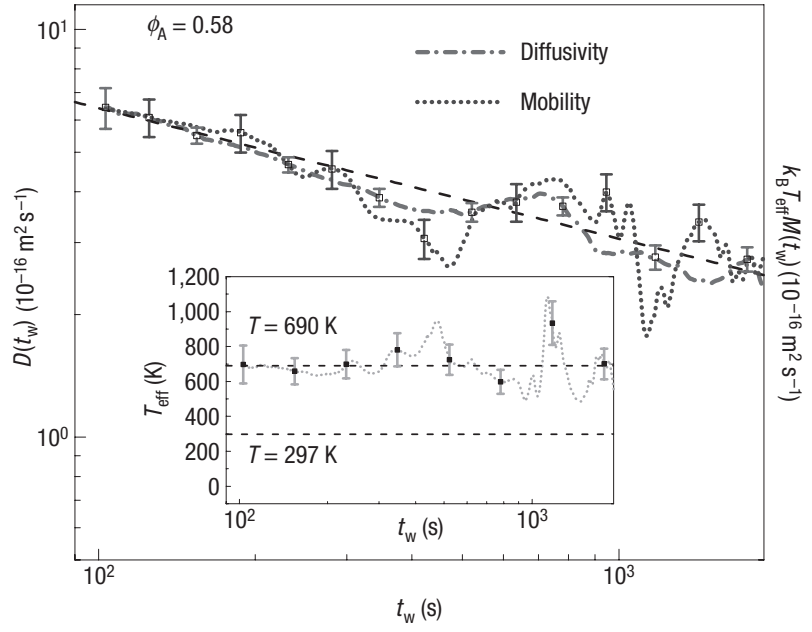

Figure 2 Diffusivity (dash-dot line) and mobility (dotted line) as a function of ageing time for sample $\mathbf{A}$. A dashed straight line is added to guide the eyes, which shows $D\left(t_{\mathrm{w}}\right) \sim t_{\mathrm{w}}^{-\gamma}$ and $M\left(t_{\mathrm{w}}\right) \sim t_{\mathrm{w}}^{-\gamma}$ with $\gamma=0.32 \pm 0.08$. For convenience of comparison with diffusivity, mobility is scaled by $k_{\mathrm{B}} T_{\text {eff }}$, with $k_{\mathrm{B}}$ being the Boltzmann constant and the effective temperature $T_{\text {eff }}=690 \mathrm{~K}$. The inset shows the effective temperature as a function of waiting time. Error bars, which are based on the statistical average and the uncertainty in locating the particle position, are only shown on some representative points for clarity.

Even though both $D$ and $M$ depend on the age of the system, their ratio is constant:

$$
T_{\text {eff }}\left(t_{\mathrm{w}}\right) \equiv \frac{D\left(t_{\mathrm{w}}\right)}{k_{\mathrm{B}} M\left(t_{\mathrm{w}}\right)}=(690 \pm 100) \mathrm{K} .
$$

The inset of Fig. 2 plots $T_{\text {eff }}$ as a function of $t_{\mathrm{w}}$. We obtain $T_{\text {eff }} \approx$ $690 \mathrm{~K}$, which is more than double the ambient temperature of $297 \mathrm{~K}$. For very large $t_{\mathrm{w}}, T_{\text {eff }}$ shows large fluctuations that are mainly due to the larger statistical error (owing to the limited number of tracers) in obtaining the mobilities and diffusivities in the large $\Delta t$ and $t_{\mathrm{w}}$ regime. It remains a question whether the long-waiting-time regime may show interrupted ageing.

Equation (2) is easy to understand when the system is at equilibrium: we extract energy from many identical tracers located in distant regions of the colloidal system and transfer it to the thermometer system. The thermometer receives work from the diffusive motion of the tracers, and it dissipates energy through the viscosity of the system. These two opposing effects make the thermometer stabilize at a temperature guaranteed by the Einstein relation. Naturally, we have applied the diffusion-mobility calculations to dilute sample $\mathrm{C}$ and find that it is equilibrated at the bath temperature (see Supplementary Information, Appendix E). On the other hand, the colloidal sample is ageing out of equilibrium. Nevertheless, the fact that the ratio of diffusion to mobility yields a constant temperature can be taken as an indication that the long-time behaviour of the system has thermalized at a larger effective temperature $T_{\text {eff }} \approx 690 \mathrm{~K}$.

Although it may seem counterintuitive that the slow relaxation at long times corresponds to a temperature that is actually higher than the equilibrium bath temperature, there is an interesting physical picture that rationalizes this observation. One can think of the energy landscape of configurations of the colloidal glass being explored less frequently, yet the amplitude of the jumps between basins corresponds to 'hotter' explorations of a broader

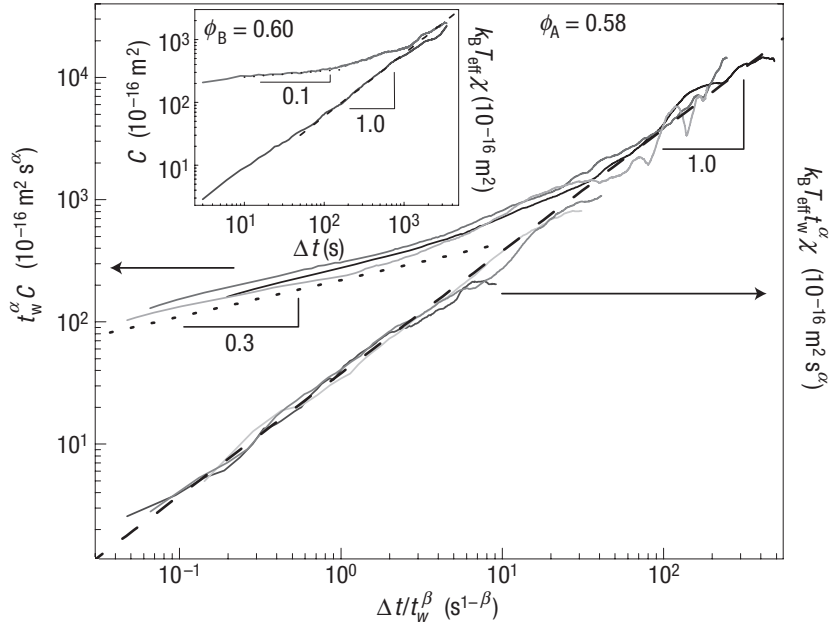

Figure 3 Scaling plot of sample A. For the scaled autocorrelation, $t_{\mathrm{w}}^{\alpha} \mathcal{C}$, and scaled integrated response, $k_{\mathrm{B}} T_{\text {eff }} t_{\mathrm{w}}^{\alpha} \chi$, as a function of the time ratio, $\Delta t / t_{\mathrm{w}}^{\beta}$, for different waiting times, $t_{\mathrm{w}}=300 \mathrm{~s}, 3,000 \mathrm{~s}$ and $6,000 \mathrm{~s}$, which all fall on the same respective curve. The dashed and dotted lines are linear fits that indicate that $T_{\text {eff }}=690 \mathrm{~K}$. The inset is a plot of sample $B$ for autocorrelation, $C$, and integrated response, $k_{B} T_{\text {eff }} \chi$, as a function of $\Delta t$ at $t_{w}=100 \mathrm{~s}$. The dashed and dotted lines are linear fits that indicate that $T_{\text {eff }}=1,600 \mathrm{~K}$ for this sample.

distribution of energy states. Although this mechanism violates the usual relations between particle motion and temperature, it gives rise to the effective temperature measured in our experiments.

\section{SCALING ANSATZ FOR THE GLOBAL CORRELATIONS AND RESPONSES}

Further insight into the understanding of the slow relaxation can be obtained from the study of the universal dynamic scaling of the observables with $t_{\mathrm{w}}$. On the basis of spin-glass models, different scaling scenarios have been proposed ${ }^{2,26}$ for correlation and response functions. Our analysis indicates that the observables can be described as

$$
\begin{gathered}
C\left(t_{\mathrm{w}}+\Delta t, t_{\mathrm{w}}\right)=\left\langle\Delta x^{2}\right\rangle / 2=t_{\mathrm{w}}^{-\alpha} f_{\mathrm{D}}\left(\frac{\Delta t}{t_{\mathrm{w}}^{\beta}}\right), \\
\chi\left(t_{\mathrm{w}}+\Delta t, t_{\mathrm{w}}\right)=\frac{\langle\Delta x\rangle}{F}=t_{\mathrm{w}}^{-\alpha} f_{\mathrm{M}}\left(\frac{\Delta t}{t_{\mathrm{w}}^{\beta}}\right),
\end{gathered}
$$

where $f_{\mathrm{D}}$ and $f_{\mathrm{M}}$ are two universal functions and $\alpha$ and $\beta$ are the ageing exponents. Evidence for the validity of these scaling laws is provided in Fig. 3 where the data of the correlation function and the integrated response function collapse onto a master curve when plotted as $t_{\mathrm{w}}^{\alpha} C\left(t_{\mathrm{w}}+\Delta t, t_{\mathrm{w}}\right)$ and $t_{\mathrm{w}}^{\alpha} \chi\left(t_{\mathrm{w}}+\Delta t, t_{\mathrm{w}}\right)$ versus $\Delta t / t_{\mathrm{w}}^{\beta}$. By minimizing the $\sigma^{2}$ value of the difference between the master curve and the data (see Supplementary Information, Appendix $\mathrm{H}$ ) we find that the best data collapse is obtained for the following ageing exponents: $\alpha+\beta=0.34 \pm 0.05$ and $\beta=0.48 \pm$ 0.05 . We find (Fig. 3 ) that the scaling functions satisfy the following asymptotic behaviour:

$$
\begin{gathered}
f_{\mathrm{D}}(y) \sim \begin{cases}y^{0.3} & y \ll 1, \\
y & y \gg 1,\end{cases} \\
f_{\mathrm{M}}(y) \sim y,
\end{gathered}
$$



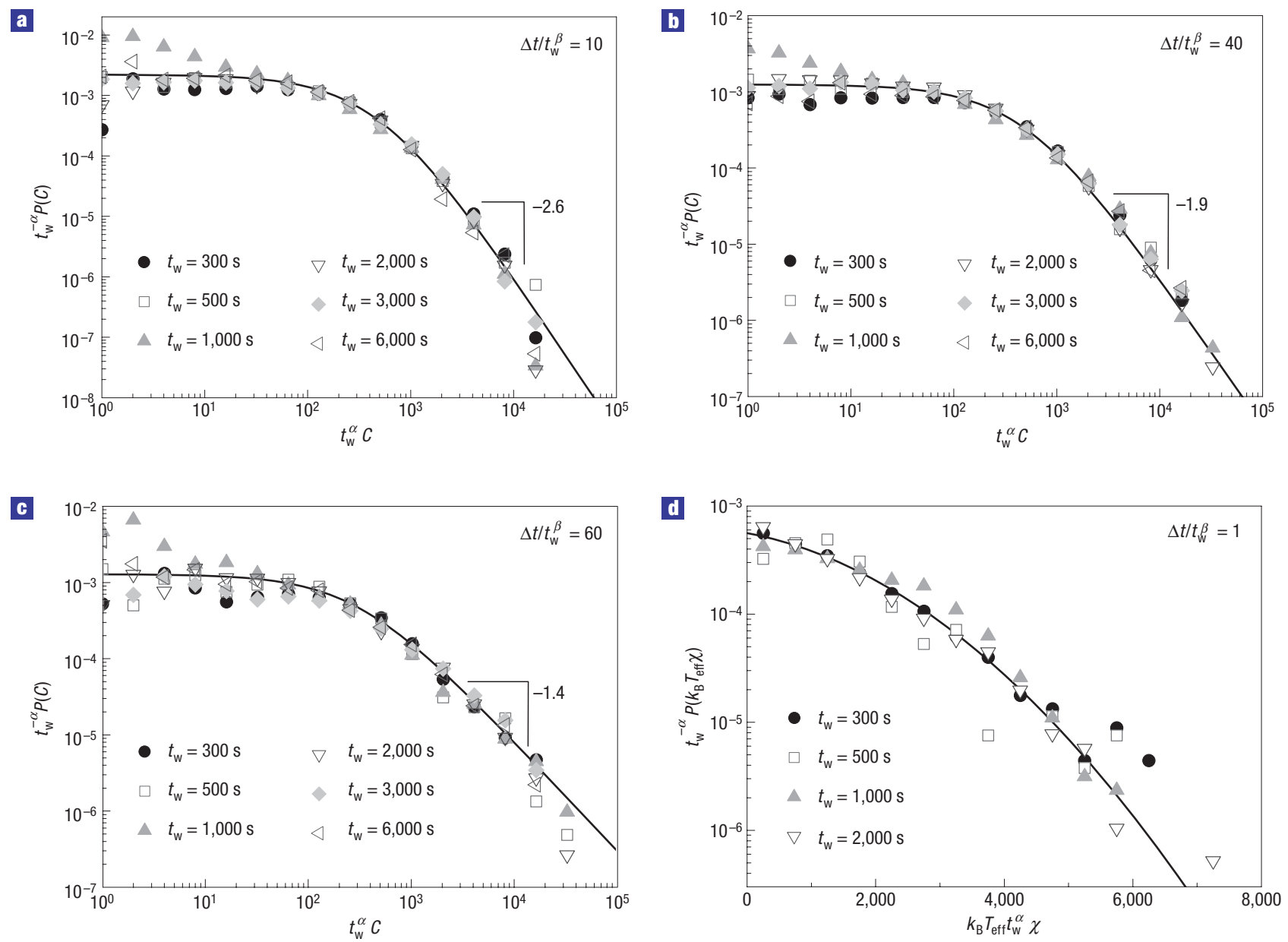

Figure 4 Study of local fluctuations. a, PDF of the scaled local correlation $t_{\mathrm{w}}^{\alpha} C$ for $\Delta t / t_{\mathrm{w}}^{\beta}=10$ with $\Delta t$ from 155 to $650 \mathrm{~s}$. The solid line corresponds to the modified power-law fit $t_{\mathrm{w}}^{-\alpha} P(C) \propto\left(t_{\mathrm{w}}^{\alpha} C+C_{0}\right)^{-\lambda}$, with $C_{0}=500$ and $\lambda=2.6$. b. Same as a, for $\Delta t / t_{\mathrm{w}}^{\beta}=40$ with $\Delta t$ from 620 to $2,600 \mathrm{~s}$. The resulting distribution can be fitted (solid line) by a modified power law with $C_{0}=500$ and $\lambda=1.9$. c. Same as a, for $\Delta t / t_{\mathrm{w}}^{\beta}=60$ with $\Delta t$ from 930 to $3,900 \mathrm{~s}$. The solid line is the best fit, with parameters $C_{0}=300$ and $\lambda=1.4$. d, The PDF of the scaled local integrated response for $\Delta t / t_{w}^{\beta}=1$ shows a gaussian decay. For the convenience of the comparison with $\mathbf{a}, \mathbf{b}$ and $\mathbf{c}$, we rescale the response by $k_{\mathrm{B}} T_{\text {eff }}$, where $T_{\text {eff }}=690 \mathrm{~K}$.

in agreement with the fact that the motion of the particles is diffusive at long times, $\left\langle\Delta x^{2}\right\rangle \sim \Delta t$, and the existence of a well-defined mobility, respectively. Therefore at long times, both the correlation and response functions exhibit the same power-law decay:

$$
\begin{aligned}
& C\left(t_{\mathrm{w}}+\Delta t, t_{\mathrm{w}}\right) \sim t_{\mathrm{w}}^{-(\alpha+\beta)} \Delta t, \\
& \chi\left(t_{\mathrm{w}}+\Delta t, t_{\mathrm{w}}\right) \sim t_{\mathrm{w}}^{-(\alpha+\beta)} \Delta t .
\end{aligned}
$$

The result $C\left(t_{\mathrm{w}}+\Delta t, t_{\mathrm{w}}\right) \sim \chi\left(t_{\mathrm{w}}+\Delta t, t_{\mathrm{w}}\right) \sim t_{\mathrm{w}}^{-0.34}$ confirms our previous result, equation (1), $D\left(t_{\mathrm{w}}\right) \sim M\left(t_{\mathrm{w}}\right) \sim t_{\mathrm{w}}^{-0.32}$. This is in further agreement with the finding that $T_{\text {eff }}$ is independent of the age of the system, $t_{\mathrm{w}}$. For short times, the MSD scaling function crosses over to a subdiffusive behaviour of the particles. We obtain, $\left\langle x^{2}\left(t_{\mathrm{w}}+\Delta t, t_{\mathrm{w}}\right)\right\rangle \sim t_{\mathrm{w}}^{-\alpha}\left(\Delta t / t_{\mathrm{w}}^{\beta}\right)^{0.3}=t_{\mathrm{w}}^{-0.004} \Delta t^{0.3}$. As the trapping time corresponds to the size of the cages denoted by $q\left(t_{\mathrm{w}}\right)$, we can determine the cage dependence on $t_{\mathrm{w}}$ as $q\left(t_{\mathrm{w}}\right) \sim t_{\mathrm{w}}^{-0.002}$. The resulting exponent is so small that we can say that the cages are not evolving with the waiting time, within experimental uncertainty. Furthermore, the scaling ansatz of equation (3) indicates that the relaxation time of the cages scales as $\tau\left(t_{\mathrm{w}}\right) \sim t_{\mathrm{w}}^{\beta}$ because this is the time for the subdiffusive behaviour to cross over to the long-time diffusive regime.

From Fig. 3 we see that the asymptotic $\Delta t$ linear regime appears when the reduced variable $\Delta t / t_{\mathrm{w}}^{\beta}>10$. The time separation between $\Delta t$ and $t_{\mathrm{w}}$ can be determined using the cutoff: $\Delta t / t_{\mathrm{w}}^{0.48} \sim 10$. For smaller times, $\Delta t / t_{\mathrm{w}}^{\beta}<10$, we obtain a subdiffusion regime (with exponent 0.3 ), which is characteristic of the cage dynamics. For longer times, $\Delta t / t_{\mathrm{w}}^{\beta}>10$, we observe the asymptotic $\Delta t$ linear regime where the diffusivity is calculated. The measurement of MSD in Fig. 1 extends up to $t_{\mathrm{w}}=6,000 \mathrm{~s}$. For this $t_{\mathrm{w}}$, the separation of timescales appears when $\Delta t>$ $10 \times 6,000^{0.48} \simeq 10^{3} \mathrm{~s}$. Our measurements for MSD extend to $10^{4} \mathrm{~s}$, ensuring a separation of timescales even for this longest waiting time. For the smaller $t_{\mathrm{w}}$ considered in the calculation of $T_{\text {eff }}$, the separation of timescales is even more pronounced. For instance, for a typical $t_{\mathrm{w}}=1,000 \mathrm{~s}$ where the $T_{\text {eff }}$ is calculated, the separation of timescales occurs at $10 \times 1,000^{0.48} \simeq 275 \mathrm{~s}$, again ensuring a welldefined long-time asymptotic behaviour for $\Delta t=10^{4} \mathrm{~s}$.

Although the existence of an effective temperature can be rationalized using theoretical frameworks of disordered 
spin-glass models ${ }^{4}$, we find that the scaling forms of the correlations and responses are not consistent with such models. On the basis of invariance properties under time reparametrization, spin-glass models predict a general scaling form $C_{\mathrm{ag}}\left(t, t_{\mathrm{w}}\right)=$ $C_{\mathrm{ag}}\left(h(t) / h\left(t_{\mathrm{w}}\right)\right)$, where $h(t)$ is a generic monotonic function ${ }^{2}$. We find that the scaling of our observables from equations (3a), (3b) cannot be collapsed with the ratio $h(t) / h\left(t_{\mathrm{w}}\right)$. The scaling with $h(t) / h\left(t_{\mathrm{w}}\right)$ is expected for a system in which the correlation function saturates at long times ${ }^{27}$. On the other hand, our system is diffusive, and the studied correlation function is not bounded. Indeed, similar scaling as in our system has been found in the ageing dynamics of another unbounded system: an elastic manifold in a disordered media ${ }^{28}$. The suggestion is that this problem and that of particle diffusing in a colloidal glass may belong to the same universality class. Furthermore, our results can be interpreted in terms of the droplet picture of the ageing of spin glass, where the growth of the dynamical heterogeneities controls the ageing.

\section{LOCAL FLUCTUATIONS OF AUTOCORRELATIONS AND RESPONSES}

Previous work has revealed the existence of dynamical heterogeneities, associated with the cooperative motion of the particles, as a precursor to the glass transition as well as in the glassy state $^{22,23,25,29}$. Instead of the average global quantities studied above, the existence of dynamical heterogeneities requires a microscopic insight into the structure of the glassy state. Earlier studies focused mainly on probability distributions of the particles' displacement near the glass transition. More recent analytical work in spin glasses $^{30}$ shows that the probability distribution function (PDF) of the local correlation $P(C)$ and the local integrated response $P(\chi)$ could reveal essential features of the dynamical heterogeneities.

Here we carry out a systematic study of $P(C)$ and $P(\chi)$ in sample A, and the resulting PDFs are shown in Fig. 4 . The scaling ansatz of equation (3) implies that $P(C)$ and $P(\chi)$ should be collapsed by rescaling the time $\Delta t$ by $t_{\mathrm{w}}^{\beta}$ and the local fluctuations by $t_{\mathrm{w}}^{\alpha}$ (see Supplementary Information, Appendix I for further details). Indeed, this scaling ansatz provides the correct collapse of all the local fluctuations captured by the PDFs, as shown in Fig. 4a,b,c for $P(C)$ and in Fig. $4 \mathrm{~d}$ for $P(\chi)$.

The PDF of the autocorrelation function exhibits a universal behaviour following a modified power law $t_{\mathrm{w}}^{-\alpha} P(C) \propto$ $\left(t_{\mathrm{w}}^{\alpha} C+C_{0}\right)^{-\lambda}$, where $C_{0}$ and $\lambda$ only depend on the time ratio $\Delta t / t_{\mathrm{w}}^{\beta}$. For the smaller values of $C\left(C<t_{\mathrm{w}}^{-\alpha} C_{0}\right)$, the existence of a flat plateau in $P(C)$ indicates that the tracers are confined in the cage. For larger values of $C$, the salient feature of the PDF is the very broad character of the distribution, with an asymptotic behaviour $P(C) \sim C^{-\lambda}$. This large deviation from a gaussian behaviour is a clear indication of the heterogeneous character of the dynamics. Furthermore, the exponent $\lambda$ decreases from 2.6 to 1.4 with the time ratio $\Delta t / t_{\mathrm{w}}^{\beta}$ ranging from 10 to 60 . We notice that $\lambda=2$ corresponds to the crossover between the short-time and long-time regime in Fig. 3, where $\Delta t / t_{\mathrm{w}}^{\beta} \approx 40$. The significance of $\lambda=2$ is seen in the integral $\int P(C) C \mathrm{~d} C$. For $\lambda>2\left(\Delta t / t_{\mathrm{w}}^{\beta}<40\right)$, the plateau dominates over the power-law tail in the integral and the dynamics is less heterogeneous. For $\lambda<2\left(\Delta t / t_{\mathrm{w}}^{\beta}>40\right)$ the power-law tail dominates and this regime corresponds to the highly heterogeneous long-time regime (see Supplementary Information, Appendix J).

In contrast, $P(\chi)$, shown in Fig. $4 \mathrm{~d}$, exhibits a different behaviour. The fluctuations are more narrow and the PDF can be approximated by a gaussian. This is consistent with the fact that we did not find cage dynamics for the global response in Fig. 1c. Moreover, numerical simulations of spin-glass models ${ }^{30}$ seem to indicate a narrower distribution as found here.

We have presented experimental results on an ageing colloidal glass showing a well-defined temperature for the slow modes of relaxation of the system. This $T_{\text {eff }}$ is larger than the bath temperature because it implies large-scale structural rearrangements of the particles. In other words, it controls the cooperative motion of particles needed to relax the cages. The interesting result is that this temperature remains constant independent of the age, even though both the diffusivity and the mobility are age dependent. The power-law scaling found to describe the transport coefficients indicates the slow relaxation of the system. A universal scaling form is found to describe all the observables. That is, not only the global averages, but also the local fluctuations. The scaling ansatz, however, cannot be described using present models of spin glasses, but it is more akin to that observed in elastic manifolds in random environments, suggesting that our system may share the same universality class.

\section{METHODS}

\section{EXPERIMENTAL DETALS}

The colloidal suspension is immersed in a solution containing $76 \%$ weight fraction of cyclohexylbromide and $24 \%$ cis-decalin, which are chosen for their density and index of refraction matching capabilities ${ }^{23}$. For such a system the glass transition occurs at $\phi_{\mathrm{g}} \approx 0.57-0.58$ (refs $1,22,23$ ). In our experiments, we consider three samples at different densities and determine the glassy phase for the samples that exhibit ageing. The main results are obtained for sample A just above the glass transition $\phi_{\mathrm{A}}=0.58 \pm 0.01$. We also consider a denser sample, $\mathrm{B}$, with $\phi_{\mathrm{B}}=0.60 \pm 0.01$, although this sample is so deep in the glassy phase that we are not able to study the slow relaxation of the system and the dependence of the waiting time within the timescales of our experiments. Finally, we also consider a sample $\mathrm{C}$ below the glass transition

$\phi_{\mathrm{C}}=0.13 \pm 0.01<\phi_{\mathrm{g}}$ for which we find the usual equilibrium relations. Before our measurements, the samples are homogenized by stirring for $2 \mathrm{~h}$ to achieve a reproducible initial time (see Supplementary Information, Appendix A for a full discussion).

We use a magnetic force as the external perturbation to generate two-dimensional motion of the tracers (see Supplementary Information, Appendix A) on a microscope stage following a simplified design of ref. 31 . Video microscopy and computerized image analysis are used to locate the tracers in each image. We calculate the response and correlation functions in the $x-y$ plane.

Received 11 December 2005; accepted 30 June 2006; published 30 July 2006.

\section{References}

1. Pusey, P. N. \& van Megen, W. Phase behavior of concentrated suspensions of nearly hard colloidal spheres. Nature 320, 340-342 (1986).

2. Cugliandolo, L. F. in Slow Relaxations and Nonequilibrium Dynamics in Condensed Matter (ed. Barrat, J.-L. et al. ) (Springer, Berlin, 2002).

3. Struik, L. C. E. Physical Ageing in Amorphous Polymer and Other Materials (Elsevier, Houston, 1978).

4. Cugliandolo, L. F. \& Kurchan, J. Analytical solution of the off-equilibrium dynamics of a long-range spin-glass model. Phys. Rev. Lett. 71, 173-176 (1993).

5. Bouchaud, J.-P., Cugliandolo, L. F., Kurchan, J. \& Mézard, M. Spin-Glasses and Random Fields (World Scientific, Singapore, 1998).

6. Kirkpatrick, T. R. \& Thirumalai, D. p-spin-interaction spin-glass models: connections with the structural glass problem. Phys. Rev. B 36, 5388-5397 (1987).

7. Bouchaud, J.-P., Cugliandolo, L. F., Kurchan, J. \& Mézard, M. Mode-coupling approximations, glass theory and disordered systems. Physica A 226, 243-273 (1996).

8. Edwards, S. F. in Granular Matter: An Interdisciplinary Approach (ed. Mehta, A.) 121-140 (Springer, New York, 1994).

9. Ono, I. K. et al. Effective temperatures of a driven system near jamming. Phys. Rev. Lett. 89, 095703 (2002).

10. Makse, H. A. \& Kurchan, J. Testing the thermodynamic approach to granular matter with a numerical model of a decisive experiment. Nature 415, 614-617 (2002).

11. Song, C., Wang, P. \& Makse, H. A. Experimental measurement of an effective temperature for jammed granular materials. Proc. Natl Acad. Sci. 102, 2299-2304 (2005).

12. Stillinger, F. H. A topographic view of supercooled liquids and glass formation. Science 267, 1935-1939 (1995).

13. Kob, W., Sciortino, F. \& Tartaglia, P. Aging as dynamics in configurations space. Europhys. Lett. 49, 590-596 (2000).

14. Israeloff, N. E. \& Grigera, T. S. Low-frequency dielectric fluctuations near the glass transition. Europhys. Lett. 43, 308-313 (1998).

15. Bellon, L., Ciliberto, S. \& Laroche, C. Violation of the fluctuation-dissipation relation during the formation of a colloidal glass. Europhys. Lett. 53, 511-517 (2001).

16. Hérisson, D. \& Ocio, M. Fluctuation-dissipation ratio of a spin glass in the aging regime. Phys. Rev. Lett. 88, 257202 (2002).

17. Buisson, L., Ciliberto, S. \& Garcimartín, A. Intermittent origin of the large violations of the fluctuation-dissipation relations in an aging polymer glass. Europhys. Lett. 63, 603-609 (2003). 
18. Abou, B. \& Gallet, F. Probing a nonequilibrium Einstein relation in an aging colloidal glass. Phys. Rev. Lett. 93, 160603 (2004)

19. Parisi, G. Off-equilibrium fluctuation-dissipation relation in fragile glasses. Phys. Rev. Lett. 79, 3660-3663 (1997)

20. Barrat, J.-L. \& Kob, W. Fluctuation-dissipation ratio in an aging Lennard-Jones glass. Europhys. Lett. 46, 637-642 (1999).

21. Berthier, L. \& Barrat, J.-L. Nonequilibrium dynamics and fluctuation-dissipation in a sheared fluid. J. Chem. Phys. 116, 6228-6242 (2002).

22. Kegel, W. K. \& van Blaaderen, A. Direct observation of dynamical heterogeneities in colloidal hard-sphere suspensions. Science 287, 290-293 (2000).

23. Weeks, E. R., Crocker, J. C., Levitt, A. C., Schofield, A. \& Weitz, D. A. Three-dimensional direct imaging of structural relaxation near the colloidal glass transition. Science 287, 627-631 (2000).

24. Megen, W. van \& Underwood, S. M. Glass transition in colloidal hard spheres: Mode-coupling theory analysis. Phys. Rev. Lett. 70, 2766-2769 (1993).

25. Courtland, R. E. \& Weeks, E. R. Direct visualization of ageing in colloidal glasses. J. Phys. Condens. Matter 15, S359-S365 (2003).

26. Henkel, M., Pleimling, M., Godrèche, G. \& Luck, J.-M. Aging, phase ordering, and conformal invariance. Phys. Rev. Lett. 87, 265701 (2001).

27. Cugliandolo, L. F., Kurchan, J. \& Doussal, P. L. Large time out-of-equilibrium dynamics of a manifold in a random potential. Phys. Rev. Lett. 76, 2390-2393 (1996).

28. Bustingorry, S., Cugliandolo, L. F. \& Dominguez, D. Out-of-equilibrium dynamics of the vortex glass in superconductors. Phys. Rev. Lett. 96, 027001 (2006).

29. Kob, W., Donati, C., Plimpton, S. J., Poole, P. H. \& Glotzer, S. C. Dynamical heterogeneities in a supercooled Lennard-Jones liquid. Phys. Rev. Lett. 79, 2827-2830 (1997)
30. Castillo, H. E., Chamon, C., Cugliandolo, L. F. \& Kennett, M. P. Heterogeneous aging in spin glasses. Phys. Rev. Lett. 88, 237201 (2002).

31. Amblard, F., Yurke, B., Pargellis, A. N. \& Leibler, S. A magnetic manipulator for studying local rheology and micromechanical properties of biological systems. Rev. Sci. Instrum. 67, 818-827 (1996).

32. Habdas, P. et al. Forced motion of a probe particle near the colloidal glass transition. Europhys. Lett. 67, 477-483 (2004)

33. Rapaport, D. C. The Art of Molecular Dynamics Simulation (Cambridge Univ. Press, Cambridge, 1995).

34. Weeks, E. R. \& Weitz, D. A. Properties of cage rearrangements observed near the colloidal glass transition. Phys. Rev. Lett. 89, 095704 (2002).

\section{Acknowledgements}

We wish to thank M. Shattuck for help in the design of the experiments and J. Brujić and S. Mistry for providing the colloidal particles and illuminating discussions. We acknowledge the financial support from DOE Division of Materials Sciences and Engineering and NSF-CMMT.

Correspondence and requests for materials should be addressed to H.A.M.

Supplementary Information accompanies this paper on www.nature.com/naturephysics.

\section{Competing financial interests}

The authors declare that they have no competing financial interests.

Reprints and permission information is available online at http://npg.nature.com/reprintsandpermissions/ 